\title{
Influence of sensorimotor experience on understanding center of gravity
}

\author{
Elise Agra ${ }^{1}$, Jason Sattizahn ${ }^{1}$, Megan Mikota $^{2}$, Sian L. Beilock ${ }^{1}$, and Susan M. \\ Fischer $^{2}$ \\ ${ }^{1}$ Department of Psychology, University of Chicago, \\ 5848 S. University Ave., Chicago, IL, 60637 \\ ${ }^{2}$ Department of Physics, DePaul University, \\ 2219 N. Kenmore Ave., Chicago, IL, 60614
}

\begin{abstract}
We investigated the effect of sensorimotor (or embodied) experience on people's understanding of the concept of center of gravity $(\mathrm{CoG})$. To create an embodied experience, we developed a set of balancing activities using a meter stick and a set of disks. Participants in the embodied group balanced systems of objects directly on their hands. Participants in the hands-on group used a fulcrum to balance the systems of objects but did not directly experience the feeling of balancing a system of objects. Participants in both groups performed better on questions in which: (1) objects are symmetric compared to asymmetric, (2) the system $\mathrm{CoG}$ is at the same location as the object CoG compared to when these locations are different, and (3) a yes/no response based on the definition of $\mathrm{CoG}$ is required compared to when the CoG location must be explicitly or implicitly located. Participants in both the hands-on and embodied groups showed an improvement in overall accuracy on questions that tested their understanding of concepts related to CoG compared to participants who did not do any balancing activities. The greatest improvement was found on the more challenging questions in which the extended objects are asymmetric, the object $\mathrm{CoG}$ is at a different location than the system $\mathrm{CoG}$, and a location related to $\mathrm{CoG}$ is explicitly requested.
\end{abstract}

\section{INTRODUCTION}

Research on physics problem solving has addressed student difficulties in understanding numerous introductory physics concepts [1-2]. However, PER studies that draw from research in cognitive science are not common. Some of the studies about the intersection of cognitive studies and physics problem solving and learning include attention, reasoning, and transfer (for an overview of these studies, see Ref. [3]). In this paper we investigate how theories of embodied cognition could be leveraged to impact learning of concepts related to center of gravity.

Embodied cognition is the idea that our mental representations of concepts involves a re-experiencing (or embodiment) of the relevant concept [4]. Expert ballet dancers experience more activation in the sensorimotor regions of the brain that are involved in dancing when they watch ballet moves than when they watch an unfamiliar dance move [5]. Similarly, when they hear descriptions of hockey-related action (e.g., "The hockey player finished the shot."), expert hockey players activate their motor systems more than novices [6].

Applying similar ideas to physics education, a recent study [7] showed that students' understanding of the concepts of torque and angular momentum improved when students physically experienced the consequences of changing angular momentum by tilting the axle of a double bicycle wheel under various configurations, compared with students who observed the exercise and received visual information. In addition, functional Magnetic Resonance Imaging (fMRI) data for participants who had direct experience feeling the associated torque instead of observing forces being exerted on someone else showed activation of sensorimotor brain areas when they later applied these concepts within a scanner [7].

The concepts of angular momentum and torque are well suited for this type of embodied activity as the spinning bicycle wheels allow students to directly experience the torque associated with the changing direction of the angular momentum vector and to easily relate what they experience to the physical quantities. But can embodied cognition methods be used to improve the learning of physics concepts such as center of gravity that map less directly onto the relevant physics equations and for which embodied experiences are not obvious?

In the current study we investigated whether people's understanding of the concept of center of gravity ( $\mathrm{CoG})$ differed depending on whether they had direct physical experience balancing a system consisting of a meter stick and a set of disks. We build off of work by Ortiz et al. [8] on static equilibrium and balancing where students showed poorer performance on questions involving extended asymmetric systems (such as a baseball bat) than for asymmetric systems that are discrete (such as two different crates balanced on a board or see saw).

\section{METHODS}

Participants were recruited from a Midwestern university community $(\mathrm{N}=101 ; 49$ male $)$. Approximately $80 \%$ of the participants had never taken a college physics class while $\sim 5 \%$ of the participants had taken three or more college 
physics classes. Each participant performed three tasks: a pre-test, a group-dependent training or control exercise, and a post-test. Each task took 20 minutes to complete.

After providing written consent and before starting the pre-test, participants were given the following definition: "The Center of Gravity is the point of balance or equilibrium of an object or a set of objects." This definition was used for simplicity to give participants who have not had any physics experience an idea of what center of gravity is for simple situations. The pre-test was a pen-and-paper task consisting of $48 \mathrm{CoG}$ questions. After completing the pre-test, participants were assigned to a control condition, a hands-on training condition, or an embodied training condition. Participants in the control condition solved a word search containing 24 words.

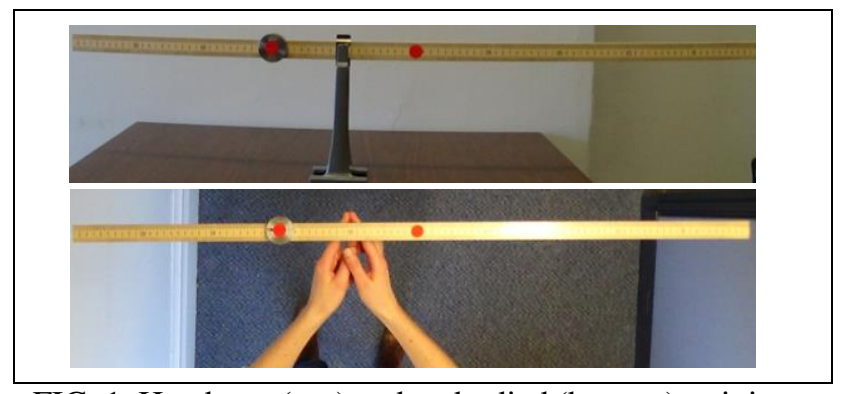

FIG. 1. Hands-on (top) and embodied (bottom) training setups. In this example, the circular disk to the left of the balance point has the same mass as the meter stick. The sticker to the right of the balance point identifies the center of gravity of the meter stick.

Training was designed to guide participants to represent an extended object as if its mass were concentrated at the location of the object's CoG. Our system consisted of a symmetric, extended object (a meter stick) and a discrete object (one of various disks). The activities were designed to lead participants to differentiate between the $\mathrm{CoG}$ of the extended object and of the system. The masses of the extended and discrete objects were in integer ratios, e.g., 1:1 or 1:2. The hands-on group balanced the meter stick and one or more hanging disks on a fulcrum (upper panel of Fig. 1), while the embodied group balanced a similar system directly on their extended fingers (lower panel of Fig. 1). A 72-question pen-and-paper post-test was completed after the training or control exercise.

The pre-and post-tests required participants to use concepts of torque and $\mathrm{CoG}$ to answer questions involving either a single extended object or a system of an extended object plus a discrete object that balanced on a fulcrum. Figure 2 shows examples of three test questions. Individual questions were categorized according to each of three characteristics: Symmetry (symmetric vs. asymmetric); Location (equal vs. unequal); Concept (application vs. definition). The extended objects were either Symmetric, such as a meter stick, or Asymmetric, such as a golf club. The CoG of the system was either at the same location
(Equal) as the $\mathrm{CoG}$ of the extended object, or at a different location (Unequal). Each question required the participant either to mark on a scale to determine a location (Application), or answer YES or NO to questions that asked if an object has a well-defined $\mathrm{CoG}$ or if the system $\mathrm{CoG}$ is at the same location as the object $\mathrm{CoG}$ (Definition). Questions for the pre- and post-tests were different, but were balanced across all categories, and were presented in the same random order for each participant.

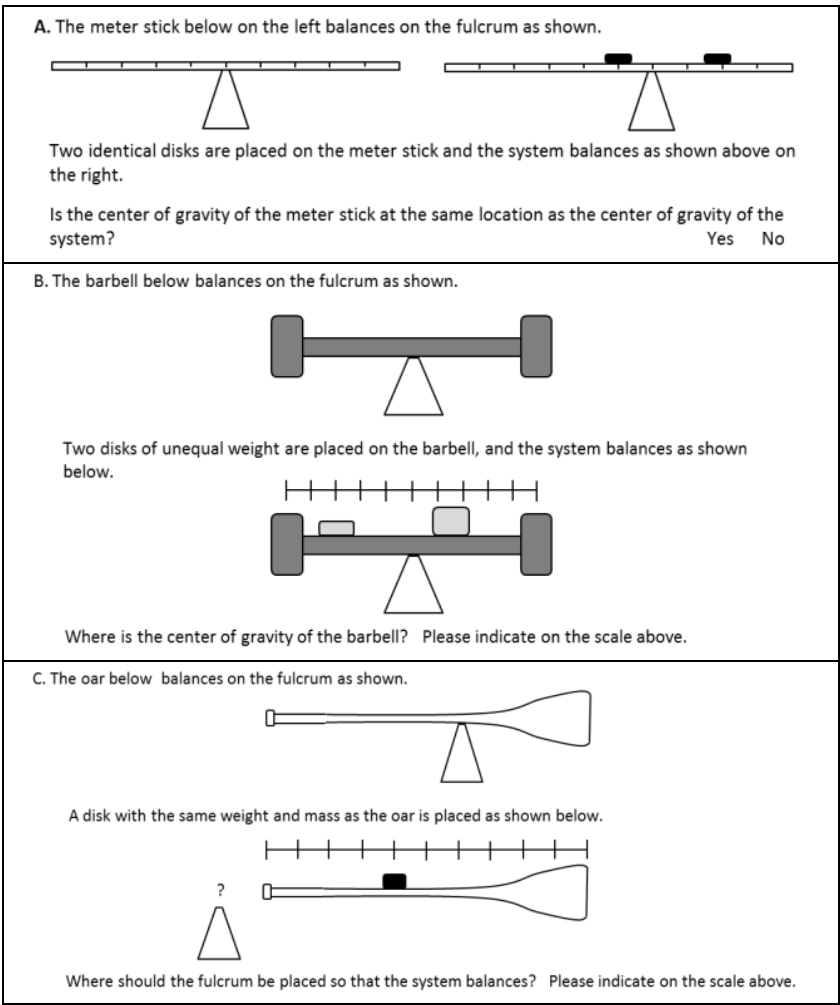

FIG. 2. Examples of questions in the pre- and post-test tasks. The top figure is categorized as DefinitionSymmetric-Unequal, the middle figure as ApplicationSymmetric-Equal, and the bottom figure as ApplicationAsymmetric-Unequal. Please see text for descriptions of the category types.

\section{RESULTS}

\section{A. Overall Accuracy}

Performance on the pre- and post-test worksheets for the control, hands-on, and embodied groups is shown in Fig. 3. Blank or unanswered questions were not scored for the analyses we present in this paper. The overall accuracy at pre-test for all groups was $\sim 0.65$. This accuracy remained approximately the same at post-test for the control group, but increased to 0.78 for the hands-on group, and to 0.83 for the embodied group.

The pre- and post-test accuracies were compared using a 3 (Condition: control, hands-on, embodied) $\times 2$ (Test: pretest, post-test) repeated measures ANOVA, which showed 
significant main effects of Condition, $F(2,98)=4.91, p=$ $.009, \eta_{p}{ }^{2}=.09$ and Test, $F(1,98)=68.01, p<.001, \eta_{p}{ }^{2}=$ .41 . These main effects are qualified by a significant Condition $\times$ Test interaction, $F(2,98)=10.17, p<.001, \eta_{p}{ }^{2}$ $=.17$. The training improved performance, but the difference in performance at post-test for the two types of training was not statistically significant. Participants in the hands-on and embodied conditions solved a significantly higher proportion of the post-test compared to the pre-test, while there was no significant difference between the pretest and the post-test for the control group.

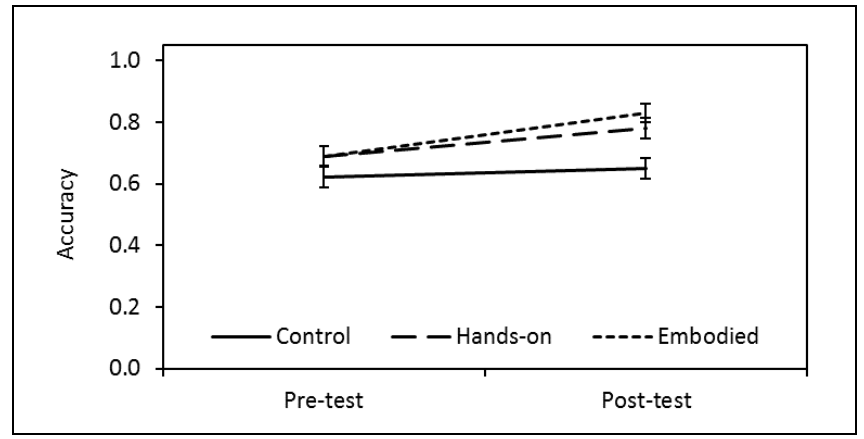

FIG. 3. Comparison of the overall pre- and post-test accuracies for the control, hands-on, and embodied training conditions.

\section{B. Training Group Performance by Question Type}

We focus next on the performance of the two training groups and consider which types of questions are most difficult and for which type of questions the training is most beneficial. Table I presents combined data for both training groups, with results for pre- and post-test performance on each of the six categories of questions.

TABLE I. Pre-test and post-test accuracy for the training groups according to the type of question.

Standard errors are given in parentheses.

\begin{tabular}{lll}
\hline \hline & $\begin{array}{c}\text { Pre-test } \\
\text { Accuracy }\end{array}$ & $\begin{array}{c}\text { Post-test } \\
\text { Accuracy }\end{array}$ \\
\cline { 2 - 3 } Symmetric & $0.75(0.02)$ & $0.83(0.02)$ \\
Asymmetric & $0.63(0.02)$ & $0.77(0.03)$ \\
Equal & $0.86(0.02)$ & $0.93(0.02)$ \\
Unequal & $0.52(0.02)$ & $0.68(0.03)$ \\
Definition & $0.85(0.02)$ & $0.90(0.01)$ \\
Application & $0.61(0.02)$ & $0.75(0.03)$ \\
\hline \hline
\end{tabular}

Perception studies in cognitive science (e.g., Ref. [9] and references within) have shown that people are much better at predicting the location of the CoG of symmetric objects and systems than of asymmetric objects and systems. Our results are consistent with prior studies, with our participants having an accuracy at pre-test of 0.75 for questions that involve a symmetric extended object being balanced compared with an accuracy of 0.63 when the extended object is asymmetric $(p<.001)$. Training, which involves balancing a symmetric meter stick, improves accuracy on questions involving both symmetric $(p<.001)$ and asymmetric $(p<.001)$ objects, with the greater gain in performance for systems with asymmetric objects.

Performance at pre-test is lowest, with an accuracy of 0.52 , on our Unequal questions, in which the CoG of the system (disk plus extended object) is at a different location than the $\mathrm{CoG}$ of the extended object. Participants train on some systems that would be categorized as Equal and other systems that would be categorized as Unequal. Equal and Unequal question types both require participants to work with concepts of torque and $\mathrm{CoG}$, but the Unequal questions also require students to differentiate between the system and extended object. Training improves performance significantly for the Unequal questions $(p<.001)$, with an accuracy of 0.68 at post-test, though this remains well below performance on Equal questions at pre-test.

Performance on the Application questions is much lower than on the Definition questions. Some of this difference can be understood by the different style of these questions. Our Definition questions were formatted as Yes/No questions, while the Application questions required participants to identify a location related to the $\mathrm{CoG}$ of the system or extended object on a figure. Training had little impact on the already high accuracy of the Definition questions, but led to an increased accuracy of the Application questions from pre-test to post-test of 0.60 to $0.75(p<.001)$. This gain in accuracy by about 0.15 was consistent for all three of the more difficult question types, whereas the gain for less challenging question types was $\sim 0.05$ (noting that participants had higher accuracy on the less challenging vs. more challenging questions at pretest).

\section{Group Difference Analysis}

As noted previously in Fig. 3, both training groups show similar increases in overall accuracy from pre- to post-test. It is possible that group differences for the hands-on and embodied participants may exist when we look at specific categories of questions. We focus on Application questions and compare the performance of the two training groups on the easiest and most challenging question types. Of the Application questions, those that involve symmetric extended objects and systems that balance at the location of the $\mathrm{CoG}$ of the object (Application-Symmetric-Equal) should be easier than those that involve asymmetric extended objects that balance at a location other than the CoG of the object (Application-Asymmetric-Unequal). The results of this comparison are displayed in Fig. 4.

As anticipated, the Symmetric-Equal questions are much easier than the Asymmetric-Unequal questions, with a participant accuracy of $\sim 0.9$ at pre-test on the SymmetricEqual questions and $\sim 0.3$ on the Asymmetric-Unequal questions. A 3 (Condition) $\times 2$ (Test) repeated measures ANOVA was conducted for both types of questions. The 
Condition $\times$ Test interaction was not significant for the Symmetric-Equal questions $\left(F(2,98)=2.77, p<.068, \eta_{p}{ }^{2}=\right.$ $.05)$, while it was significant for the Asymmetric-Unequal questions $\left(F(2,98)=9.70, p<.001, \eta_{p}{ }^{2}=.17\right)$. Table II shows the results of a repeated measures ANOVA comparing the pre-test and post-test accuracies of these groups for these questions. Both the hands-on and embodied groups improve from pre- to post-test on both types of questions, but improvement is greater on the more difficult questions. The effect size for the embodied group is twice the effect size for the hands-on group on Asymmetric-Unequal questions, suggesting a stronger effect (though these group differences are not statistically significant).

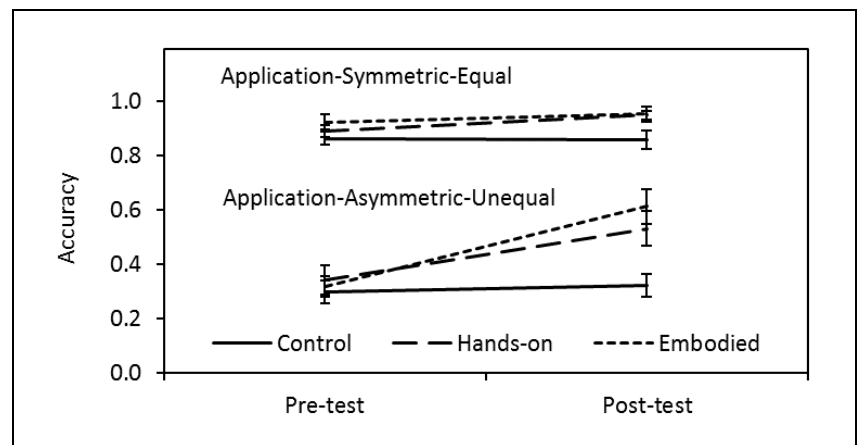

FIG 4. Accuracy by condition of Control, Hands-on or Embodied group on pre- and post-test questions that were categorized as either Application-Symmetric-Equal (top) or Application-Asymmetric-Unequal (bottom).

\section{IV.CONCLUSIONS}

In this study, we investigated the effects of two training experiences on people's understanding of the concept of center of gravity. When participants performed a set of guided balancing activities using a meter stick and a set of disks, either by using a fulcrum or their own hands, the participants' overall accuracy on center of gravity problems improved. The lowest accuracies at pre-test and the greatest improvement at post-test were observed for questions where the balanced object was asymmetric, the system $\mathrm{CoG}$ was at a different location from the extended object $\mathrm{CoG}$, and participants were asked to identify the balance point, or the location of the fulcrum or disk that would allow the extended object to balance. Prior research comparing embodied experience with observational experience in learning concepts related to angular momentum suggests that the gain in learning specifically due to embodied activities may be observed only for these more challenging questions [7].

TABLE II. Results of a repeated measures ANOVA comparing the pre-test and post-test on Application Symmetric-Equal (SE) and Asymmetric-Unequal (AU) questions for the hands-on and embodied groups.

\begin{tabular}{llc}
\hline \hline \multirow{2}{*}{ SE } & Hands-on & No significant Condition $\times$ Test \\
\cline { 3 - 3 } & Embodied & \multicolumn{1}{c}{ ANOVA Result } \\
\multirow{2}{*}{ AU } & Hands-on & $F(1,98)=17.24, p<.001, \eta_{p}{ }^{2}=.15$ \\
& Embodied & $F(1,98)=42.27, p<.001, \eta_{p}{ }^{2}=.31$ \\
\hline \hline
\end{tabular}

Several factors pose challenges for making a clean comparison between the hands-on and embodied experiences in this study. While it is true that a system will balance at its $\mathrm{CoG}$, the experience of balancing maps indirectly onto the concept of $\mathrm{CoG}$, particularly in comparison to the angular momentum study that used a system of double bicycle wheels. As a consequence, participants in both of our training groups required substantial verbal description from the experimenter in order for the training to make sense. The verbal descriptions, which were equivalent for both training groups, may have dominated the training experience, in a sense over-training the participants and weakening group differences. In addition, the pre- and post-tests included a significant fraction of questions (e.g., ApplicationSymmetric-Equal questions) that participants were able to answer with high accuracy $(>0.85)$ at pre-test, and for which the training had a fairly small, positive effect (possibly indicating a ceiling effect). Future studies will reduce the amount of verbal description given to participants in the training groups, and will include a greater proportion of questions that are in categories we have found to be the most challenging for students.

\section{ACKNOWLEDGMENTS}

This material is based upon work supported by the National Science Foundation under Grant No. 1348614
[1] D. Hestenes, M. Wells, and G. Swackhamer, The Physics Teacher 30, (1992).

[2] L.C. McDermott, P.S. Shaffer, and the Physics Education Group at the University of Washington, Tutorials in Introductory Physics (Prentice Hall, New Jersey, 2002).

[3] J. L. Docktor and J. P. Mestre, Phys. Rev. ST: PER 10, 020119 (2014).

[4] P. M. Niedenthal et al., Science 316, 1002 (2007).
[5] B. Calvo-Merino et al., Cerebral Cortex 15, (2005).

[6] S. L. Beilock et al., in Proceedings of the National Academy of Sciences, 2008, pp. 13269-13273.

[7] C. Kontra et al., Psychol. Sci. 26, 6 (2015).

[8] L. G. Ortiz, P. R. Heron, and P. S. Shaffer, American Journal of Physics 73, 6, (2005).

[9] J. Sattizahn et al., Mind, Brain, and Education 9, 3 (2015). 